\title{
Blockchain Technology Facing Socioeconomic Challenges. Promise versus Probability
}

\author{
https://doi.org/10.21272/sec.3(4).13-24.2019
}

Belen Suarez Lopez, ORCID: https://orcid.org/0000-0002-6323-2621

Reacción Economica, Lead Economist Researcher and CEO; ISO56008 Member Expert for Innovation Measurements at ISO - International Organization for Standardization, Spain

David Issó García, ORCID: https://orcid.org/0000-0002-3815-8140

PhD Researcher in Sociology, Associate Professor, Faculty of Educational Science, Granada University, Spain

Antonio Vargas Alcaide, ORCID: https://orcid.org/0000-0002-9791-2377

Economist Research, Project Manager Professional at Blockhub, Spain

\begin{abstract}
This paper has the main purpose to make a critical and balanced analysis about the potential of blockchain technology to face some of the great current socioeconomic challenges, being focus on impact assessment point of view, analyzing the disruptive potential of blockchain to provide solutions at level of different challenges as example, climate change, migrant movements, gender equality, financial inclusion or the cost opportunity of the management of data science. The term blockchain summary a numerous different type of system of distributed ledger, essentially, it is just a record distributed, a ledger of digital events that is distributed or shared among many different parts within an ecosystem (nodes), and chronological in a network. The technology is at an early stage and can be implemented in many ways depending on the objective. The methodological tool for the research is strategic and qualitative SWOT analysis identifying the critical success factors such internal factors (Strengthens and Weakness), and external factors (Opportunities and Threats), summarizes the arguments and counterarguments within the scientific discussion. From the bibliographic review carried out on the finding and disclosure provided by empirical research about business case studies, the research results summarized in the paper confirm that although looks difficulty of give a closed definition to variety of system under the umbrella of blockchain, among the main strengths of technology are its intrinsic characteristics, such as, its ability to store data immutably without relying on a central authority. As weakness, highlight the fact of the need of solve some non-minor inefficiencies as energy consumption and, as result, the difficulty to be scaled. It has the potential to replace the intermediary and central entities or change the way they works, allowing disintermediation and potentially empower people in trade, democratic participation, social interaction and financial inclusion, which represent great opportunities. Although, on the side of threats there is lack of knowledge about the technology, which generates resistance from regulators who are beginning to assess risks and are concerned about how new participants could cannibalize their income models. In addition, it seems clear the importance of assume the fact that the technological changes take time to develop and often require the adaptation of entire ecosystems.
\end{abstract}

Keywords: blockchain, decentralization, democratization, financial inclusion, socioeconomic challenges, tokem traceability, transparency, trust.

\section{JEL Classification: E4.}

Cite as: Belen Suarez Lopez, David Issó García, Antonio Vargas Alcaide (2019). Blockchain Technology Facing Socioeconomic Challenges. Promise versus Probability. SocioEconomic Challenges, 3(4), 13-24. https://doi.org/10.21272/sec.3(4).13-24.2019.

(C) The Authors, 2019. This article is published with open access at Sumy State University. 


\section{Introduction}

Seeing beyond the main trend of Blockchain hype, it is necessary to reach an objective and a strategic analysis, from which we are able to build practical solutions to our socioeconomic challenges avoiding the lack of knowledge or the fear to the unknown. At the same time, the opportunity cost of seeing the disruptive technologies, such as, Blockchain technology, Internet of Things (IoT) or Artificial Intelligence (AI). Also, provide a key approach from which to assess the value propositions of this technology.

The disruptive potential of this type of Distributed Ledger Technology (DTL) to change the organizations way and capture value is very promising, although there are important challenges to face in the implementation phase. Those who venture that the analogy of Blockchain will do for transactions what the internet did for information. Being an emerging technology, there is a lot of confusion about it and the opinion, in certain cases, is very polarized (Michelman, 2017). On one hand, we find the defenders, who value the high potential of this innovative technology and focus on highlight the benefits including their possibility to change the way organizations operate, the benefits in terms of reducing the cost of verifications or the opportunity to reduce the market power of intermediaries in favor of peer transactions, decentralizing market power and generating wide margins. On the other hand, detractors specify current limitations (for instance, in terms of inefficiencies of energy consumption or the low number of transactions per second), and focus only on the borders of cryptocurrency applications. In addition, this lack of knowledge, generates resistance from regulators who are beginning to assess risks and are concerned about how new participants could cannibalize their income models. Among the risks for the technology, the potential threat that quantum computing could represent shouldn't be ignored. In any case, its characteristics, once solved some critic inefficiencies, have a high potential to make valuable contributions.

Blockchain technology is an approach that has the potential to enhance decentralization, transparency, equality and accountability in internet. What makes this technology unique is its ability to store data immutably without relying on a central authority; it has the potential to replace the intermediary and central entities with a code that can reliably connect users with each other (Tapscott and Tapscott, 2016). The fundamental common characteristic that all Blockchain based services share is a design that depends on immutability and decentralization in storing data. The applications of Blockchain technology are numerous since they allow disintermediation in ways that can potentially empower people in trade, expression, democratic participation, social interaction and financial freedom.

Starting from the difficulty of giving a closed definition to variety of system under the umbrella of Blockchain, this research make a holistic insight about the key features, such as the reduction of cost of verification, privacy, how to solve the last mile problem, tokens and the possibility to create extensive margins, programmable currencies and the use of smart contracts.

\section{Literature Review}

This paper summarizes the arguments and counterarguments within the scientific discussion on the issue. Due to the technology is in a initial state of development, the literature and empirical studies on the impact of the applications are still in the execution phase, so it's now when applications begin to develop in numerous fields. Therefore the absence of numerous scientific literature has been complemented with articles and research from technology developers and dissemination provided by the research on case studies.

The literature review can be classified in three main blocks:

1. First block, referring to the complex definition of the limits of technology, and an overview about it. Information that allows us to understand the diversity of systems in the Blockchain world and, at the same time, analyze the essential common characteristics of the systems, which represent the key to evaluate the true value proposition of this technology and anticipate its potential for socioeconomic impact.

2. Second block, regarding the challenges themselves, so from the understanding of the problem root causes, be able to carry out an assessment of the hypothetical impact of blockchain developments.

3. Third block, referring to the weaknesses that must be solved and the challenges to overcome in order to be able to display the full disruptive potential of this general purpose technology. 


\section{Methodology}

Due to the limitations related to an emergent technology, being in the embryonic phase the most of the applications developed and adding to the fact that Blockchain refers to a set of systems and platforms, this scientific research has been based on a qualitative analysis, which should be confirmed by subsequent empirical analysis. The methodological tool for research is the strategic and qualitative SWOT analysis that identifies critical success factors, such as internal factors (Strengths and Weaknesses) and external factors (Opportunities and Threats). The research is developed from the next hypothesis:

Hypothese 1: The set of technologies under the Blockchain umbrella could potentially enable solutions to solve current socio-economic challenges.

Hypothese 2: Besides the challenges facing vulnerable groups, it exists the difficulty to defend their rights and the limited access to resources.

Then, the limitations of defending their rights in accordance with laws and the difficulty access to economic resources, to basic services, identity, ownership and control over land and other forms of property, inheritance, natural resources, appropriate new technology and financial services, including microfinance, all of them are a critical key point for these groups.

Hypothese 3: To empower and promote the social, economic and political inclusion, so it is necessary to deliver transparent, effective, credible, accountable and legitimate economic and financial structures.

Hypothese 4: Sustainable management and efficient use of limited resources need to strengthen the scientific and technological capacity.

The scientific and technological capacity represent the key to move towards more sustainable patterns of consumption and production while avoiding inefficiencies in the systems and encourage wasteful consumption by removing market distortions.

From the systematization literary sources and approaches for solving the problem, the investigation starts from the framework of the technology definition. Its novel features would be the reduction of cost of verification, the disruption into the level of privacy, the vehicular use of tokens through the platforms, the potential use of smart contracts, and the advantages of using programmable money. Then, each socio-economic challenge proposed is studied, such as the climate change, migratory movements, gender equality and management of scientific data, in order to analyze how the technology could solve it.

The framework used to establish the economic challenges object of the research has been the Sustainable Development Goals (SDG) of the UN 2030 agenda (General Assembly, United Nations, 2017). The scope of the objectives has been summarized, in order to provide a simplified and representative core root causes framework, developing the research focused on these challenges: climate change, migratory movements, gender equality and scientific data management.

\section{Results}

In order to make the qualitative analysis, the research begins from the difficulty to give a concrete definition of the set of system under the umbrella of Blockchain.

\subsection{What is Blockchain}

Blockchain, essentially it is just a digital record, a digital event which is distributed or shared among many different parts within an ecosystem (nodes), so it is distributed. It can only be updated based on the consensus of the majority of the participants in the system, and once the information has been entered, it cannot be deleted (or, at least, easily deleted). It is therefore immutable. The ledger is often secured through an intelligent combination of cryptography and game theory, and does not require reliable nodes such as traditional networks (Church, 2017). It can be verified by third parties, so it is reliable. The records may contain different types of shared data: transaction records, document attributes, credentials or other data. This information is stored chronologically in chained blocks, so each chain contains an accurate and verifiable record of the modifications that have been made in its history.

If any of the nodes were compromised, the blockchain would still work. It is necessary that more than half of the nodes were affected to compromise the network. This issue is called a "51 per cent attack". Because every node validates a transaction before it gets added to the blockchain, it is very difficult to duplicate or fake 
transactions. For the same reason, it is also virtually impossible to modify previously stored transaction data. This kind of data storage does not supported by one centralized server. Therefore, blockchain data is not susceptible to hacking and hardware damage by the way those systems are. The term Blockchain concerns a numerous different types of networks and consensus algorithms, which understanding is crucial to focus on the perspective of business innovation and applications.

\section{Types of networks}

In a Blockchain-based system, the participants are geographically distributed and connected via different kinds of networks, which can be classified into two main types: permissionless and permissioned (Baliga, 2017).

In a permissionless system or open-ended systems, any node can conduct transactions as well as take part in the consensus process to advance the Blockchain. The number of nodes is expected to be large, and these nodes are anonymous and untrusted since any node is allowed to join the network. Consensus mechanisms for such setup have to take into account for maliciousness.

Permissioned platforms are aimed at consortiums where participation is close-ended. While clients are allowed to submit transactions, the Blockchain is restricted to a fixed set of peering nodes that are run by consortium members. Permissioned platforms have semi-trusted members, in which only known participating nodes that are part of a consortium, are verified and registered. These groups are expected to be small in number and therefore can employ alternative consensus mechanisms to those used by permissionless networks.

\section{Type of Consensus Algorithms}

Regarding Blockchain, a consensus algorithm could be defined as a process, encoded by software, where computers in a network or nodes reach an agreement about a set of data, which is used to agree on a block of transactions that should be appended to the blockchain.

This is a key concept, because a Blockchain based system is as secure and robust as its consensus model (Baliga, 2017). We can find different types:

A. Proof of Work (PoW). This is the algorithm that runs Bitcoin, Ethereum, and many other networks. In the PoW, the nodes are like "miners". Miners solve mathematical equations, or hashes, and add blocks to the Blockchain. The first miner to solve a hash adds the block to the chain and is rewarded with "mined" crypto tokens, incentivizing miners to work to secure the system. The PoW algorithm consumes enormous resources and wealthy players hoard the expensive mining technology, so they gain too much influence for a network to be truly decentralized. PoW has a massive environmental problem, because of this they are difficult to escalate.

B. Proof of Stake (PoS). Nodes "forge" new blocks of transactions. In PoS, these "forgers" must "stake" their coin if they want to create a new block. When coins are staked, they are locked into a bonded wallet via smart contract and are susceptible to slashing (i.e., losing some coins) if faulty blocks are created by a forger. This consensus algorithm has a more stabilized token value and less environmental impact; this is because PoS does not require its nodes to drain as much energy for complex hashing math. It is more environmentally friendly than PoW.

C. Others, as Proof of Elapsed Time, Byzantine Fault Tolerance and variants, or Federated Byzantine Agreement. Giving an example we can refer to Casper, which is a Hybrid between PoW and PoS.

The combination of systems and consensus algorithms determines the different Blockchain Protocols, which currently have different degree of implementation, being Ethereum the one that seems to have the highest degree of penetration according to the study Stanford Graduate School of Business / Blockchain for Social Impact, 2019.

Depending on the challenge we face, it will be necessary to analyze the characteristics of each protocol to select the one that best suits each specific case.

\subsection{Blockhain Characteristics, Strengths and Weaknesses}

Despite the diversity of Blockchain technology systems, most of them have a number of common characteristics, such as their ability to reduce verification costs, privacy treatment, the need to resolve the last mile problem, the use of tokens and, in some cases the possibility of developing smart contract. 


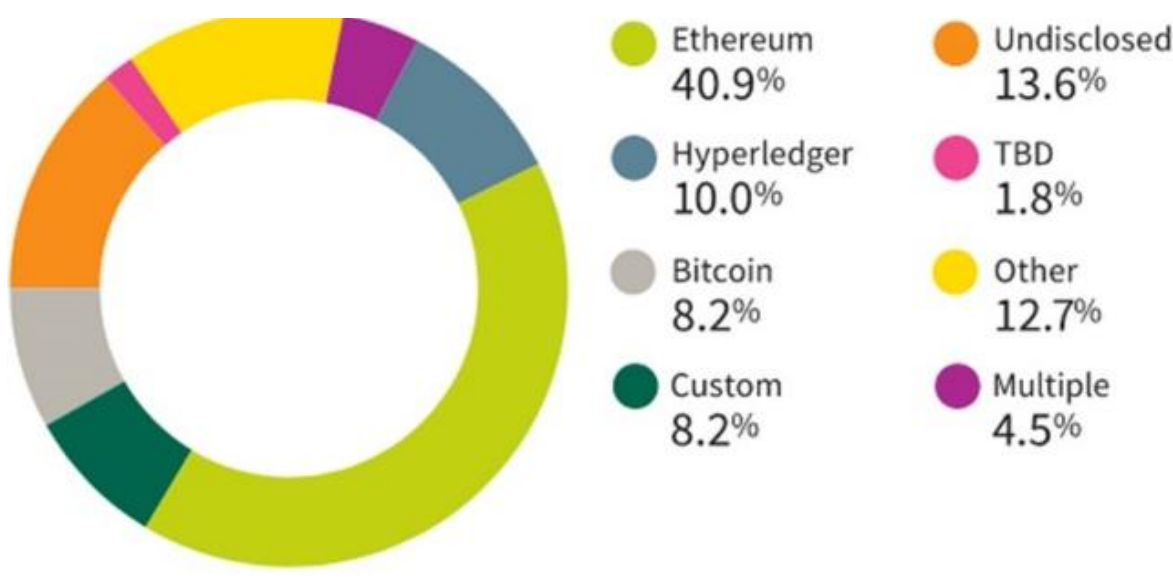

Figure 1. Type of blockchain used

Notes: Ethereum was most common among our sample, but the landscape is fragmented. $\mathrm{N}=100$. Source: Stanford Graduate School of Business/Blockchain for social impact.

\section{Reduction of the Cost of Verification}

All businesses and organizations participate in many types of transactions every day. Each of those transactions requires a different kind of verifications. In many cases, that verification is easy. Let's think in the case when you know your customers, their customers, their colleagues and their business partners. After having worked with them and their products, data or information, you have a pretty good idea of their value and reliability. But when a problem arises, it's necessary to do some type of verification or audit, either by executing some internal verification process, or to hire services from external auditors. This is an expensive and laborious process for the society and, as a result, the market slows down and has to incur in additional costs to match demand and supply (Catalini, 2019).

The reason why distributed accounting books become so useful in these cases, is because allows always the possibility to come back to the blockchain and check them. It's a free verification.

Transaction

is born

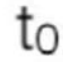

\section{Attributes}

e.g., existence, timestamp, parties

involved, conflict resolution rules, collateral etc.

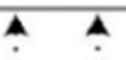

؛

$\vdots$

costly verification through an intermediary (audit)

Actions are

performed

$t_{1}$

Reliance

on transaction

attributes
Problem

may arise

$t_{n}$

Verification

of attributes

is required

\section{costless verification on a blockchain}

Figure 2. Costly veification through and intermediary (audit) versus costles digital verification on a blockchain

Source: Catalini and Gans (2018).

Because blockchain verifies reliability, it has the potential to replace intermediate and central entities to connect users to each other (Catalini and Gans, 2018). Therefore, the friction of the transaction is reduced, resulting in cost and time saving. The Internet has already allowed a faster exchange of goods and services. But intermediaries are still needed, which is expensive concerning expenses from processing payments, maintaining a reputation system, and matching demand and supply. Here is where Blockchain technology, 
combined with a crypto token, allows to rethinking a complete value chain from scratch. Obviously, it represents a threat to the current intermediaries.

\section{Privacy}

Contrary to what we may initially think when we talk about a ledger distributed with all the information and among all the nodes in the ecosystem, Blockchain offers important advances related to our privacy. Blockchain technology could mean more privacy and security avoiding data leakage (Catalini, 2019). For example, in case of a customer showing his passport or driver's license, just to demonstrate his age, the document is revealing much more. All actors disclose private information at every step of the way. The private data is replicated in a number of different providers, data silos and anywhere.

Information disclosure is increasingly becoming a cost due to data breaches. One solution could be provide a model in which would be possible verify that certain attributes are true or false, using a decentralized infrastructure without revealing all these attributes all the time.

So, blockchain offers an important opportunity to verify certain accurate information without necessarily revealing other underlying information, it carries out a selective disclosure to reveal to the appropriate interlocutor only the precise information and keep anything else private. We could talk about layers of information from which, the data owner gives access to third parties according to the concrete need, avoiding data leakage.

This technology enables new ways to manage the ownership of our data in the digital world, allows rethinking of fundamental concepts such as identity and authentication by returning control of the data and establishing more precise controls and benefiting from the advantages of sharing information avoiding hazards.

\section{The Last Mile Problem}

The last mile problem is a necessary obstacle to solve in order to realize the full potential of Blockchain. It refers to how to connect transactions between the online world and the offline world, that is, the interface between the offline world and the digital record of the event that occurs offline and how to close the gap between the two. It is necessary some mechanism or intermediary that brings confidence to the verification of that fact (Barrera, 2019). We talk about devices or people who function as oracles capable of building and feeling things in the real world and recording them without being manipulated. At this point, Blockchain joins its potential to Internet of Things (IoT) devices, providing real-time information.

Considering that we are going to need an intermediary, it is essential its reliability. Its objective is to contribute with trust and integrity information, its contribution of value to the ecosystem it would be good to evaluate risks and the frictions. This contribution could be rewards with the use of tokens.

\section{The use of Tokens}

Two typical functions of tokens are transaction settlement and indication of ownership rights. While these are the most common functions for tokens, there are varieties of other tokens ways according to the design or the goals of each platform. A settlement token facilitates the exchange of a particular product or service for money (including cryptocurrencies). Bitcoin itself is typically used to settle transactions; in fact, mining rewards can be considered a transaction in which the miners provide computing power to secure the network and are remunerated with Bitcoin in return. A platform participant contributes with time or resources and is paid for that contribution.

Being a type of distributed ledger, it is very useful for tracking ownership of assets over time, and, therefore, for facilitating trade of those assets and transactions involving other products and services. Each token is unique and identifiable, so permits track the use of each specific token.

From the social impact assessment point of view, must be highlight that the power to promote transactions is conditioned by the volatility of value of this token. For instance, in the case that a Blockchain platform has the mission of social or financial inclusion, it could be crucial that the token of reference are not volatile, and keep stable the value to assurance the social demands. However, using Blockchain as the foundation for currency and exchange, programmable currencies offer the opportunity to align the individual interest (for example, a digital currency that can be used to acquire a new product or service) with broader, more holistic community interests (the digital currency can only be accepted by organizations that engage in, or meet, some desirable practice or standard). 
The novelty is the programmable money, from minute one of its creation. Therefore, we think of being able to write contracts, which will act under certain conditions and react to market conditions or incentives. In the scope of Central Banks, talking about programmable money could mean a powerful control mechanism in terms of Monetary Policy, being each token-cryptocurrency unique, identifiable and programmable. The fact of being programmable enables the possibility that in its design it could be adapted to the circumstances of the moment breaking the inflexibility barriers demonstrated during the recent economic crises, so in times of recession, perhaps temporally oxidation mechanisms could be introduced in a programmable cryptocurrency with the goal to stimulate the exchanges.

\section{Smart Contracts}

Smart contracts are a piece of code that, once stored on the blockchain, does their task autonomously. For example, a smart contract could automatically pay a musical artist each time their song is played on the radio.

Due to its open nature, and due to the fact that the contract code is known in advance and it cannot be changed or altered by malicious actors, many experts suggest that smart contracts could replace traditional contracts. Smart contracts are fast, they execute itself immediately and autonomously and there is no possible influence it (Petersson, 2018).

There are many fields that can be disrupted by smart contracts: voting, management, supply chains, healthcare and real estate. The ability to have several parties cooperating without any of them being able to manipulate or use the system in their favor, being unhackable and transparent not only paves the way for new innovations and collaboration between bodies not possible before, as a result the development of extensive margins, but also makes those procedures much more efficient.

However, there is also resistance because now, the user should to study the code and understand programming language. Few users have the knowledge to do so and fewer could spend the time to go through it. Those who reject smart contracts argue that this system is not "trustless" after all, highlighting the inherent risks such as failures in the programming can escalate problems. Once the technology had been defined and its characteristics studied, then possible applications in different fields as climate change, migration movements, gender equality or the management of data science, will be assessed.

\subsection{Blockchain facing Challenges. Opportunities and Threats}

Following the logical framework approach, we analyze below how Blockchain platforms can help to face important socio-economic challenges. The methodology will be beginning with the synopsis of the social problem and later evaluate how Blockchain can work on the solution.

\section{Climate Change}

Climate change represents the most important milestone of our generation. The impact caused by the human race on the planet since the Industrial Revolution is such that we have inaugurated the Anthropocene period (Issberner and Léna, 2018), finding ourselves at the beginning of the sixth mass extinction of species (one in eight is currently at risk of disappearance). The temperature of the planet undergoes a vertiginous ascent, which predictably could soon be irreversible, as a result, among others consequences, very populated cities are gradually sinking with very serious consequences for their population, increasing frequently extreme weather events in addition to fires and droughts.

Within possible solutions to this great challenge, we can mention the transition to renewable energies, the search for a greener industry, the construction of habitable cities, work for the reforestation of forests or encourage the use of zero-emission transport; all of them, actions related to the objectives of the Paris Climate Conference, 2015, where 195 countries established a global action plan that puts the global warming limit below of $2^{\circ} \mathrm{C}$.

Blockchain could be a powerful tool to combat climate change. According to the Distributed Ledger Technology (DTL) for Climate Action Assessment, 2018 report, "While climate change is a truly global problem, it is well recognized that it requires a decentralized, multi-stakeholder, bottom-up approach to be solved, along with an open and transnational platform of existing (carbon markets, taxes, pricing) and evolving climate action instruments (Nationally Determined Contributions). Furthermore, the approach needs to allow for a high level of measurement, reporting, and verification (MRV) and enforce high levels of trust and transparency. DLT is a technology that optimally aligns with these requirements" (Born, 2018). 
So, Blockchain already study characteristics of decentralization, democratization and digitization, which offer great potential for the fight against climate change (Stanford Graduate School of Business / Blockchain for Social Impact, 2019).

Decentralization facilitates more efficient asset operations, decreasing the market power of intermediaries, focusing on increasing the proportion of renewable energy sources, better management, and smart consumption, contracting and storage. As a result, the distribution of energy can be accelerated.

Democratization favours peer exchange, increasing the market dynamism. Enables micro energy optimizations at the facility level by allowing people to track their energy use through a transparent and easily accessible platform.

Digitization increases traceability and allows minimizing the opportunity costs by increasing the use of underutilized capacity. Generates a reliable and effective registry, which could open the door to the development of crowdfunding projects by being more transparent and traceable the use of the funds provided. Effective evaluation, reporting and verification are essential for decision-making and evaluation of actions, all of these encouraged by the technology.

Rewarding of decisions by the use of token and ecological cyptocurrencies, it encourage consumer to take efficient and positive decisions to fight the climate change. At the same time provide liquidity and helps the creation of new forms of green financing. For instance, the tokens could reward consumers for reducing emissions of carbon. Users could earn redeemable tokens that they can get by making everyday choices to keep theirselfs energy efficients or positive climate behaviours, such as recycling and conscious consumption. The tokens can also modify emissions trading between companies and issuers, providing a transparent carbon credit market, and therefore reduce the cost of the compensation process.

\section{The Immigrant Movements}

Giving a synopsis of the issue and according to the UN Refugee Agency, in 2018 a daily average of 37,000 people were forced to flee their homes. Worldwide, the number of people fleeing persecution, conflict and violence has reached 70 million for the first time, between their, Children represent approximately half of the world's refugee population (Global Trends Forced displacement in 2018 report, 2019).

Difficulties in the field of identity (such as the situation of undocumented immigrants), abuses by organizations (such as third-party agents), financial exclusion and lack of access to basic goods are some of the problems facing millions of people in the world today.

Blockchain offers applications to solve numerous challenges faced by immigrants, governments, NGOs, and technologists. Real cases are concerning to facilitate identity and financial inclusion, providing traceability and allowing migratory movements to be more transparent and fair through:

1. Allows accessibility to digital identities and other essential documents. Having a digital identity is critical. Storing a digital version of a passport, driver's license or a user's government ID means that the individual person can always prove their identity. The digital identity also allows the government of the host country, to have a greater amount of information per period, improving the ability to evaluate people through extensive criminal background checks and medical care, for example. Greater transparency also means more responsibility for both the immigrant and the host country government, thus avoiding situations of abuse of power. Blockchain enables digital identity by making our world to be more transparent, secure and immutable in the future.

2. Creating instant payment methods and facilitating financial inclusion. Millions of workers around the world make daily money transfers to their countries of origin, having to bear high transfer costs that can take days to complete. The use of mobile wallets facilitates financial inclusion by providing access to finance where it is often impossible to have access to a traditional bank account. The new mobile payment infrastructure is also feasible with a Blockchain mobile wallet in order to pay for basic goods and services such as food.

3. Traceability in donations and crowdfunding. Blockchain allows the crowdfunding process to be transparent, secure and accessible by being verifiable by third parties. The access and monitoring open the door for donors who want to contribute to the welfare of refugees worldwide, preventing theft and generates a greater impact of the funds destined to the cause. Despite being at an early stage of introduction, it has been empirically shown that the success rates of crowdfunding platforms that use Blockchain technology are often higher than those that do not. 


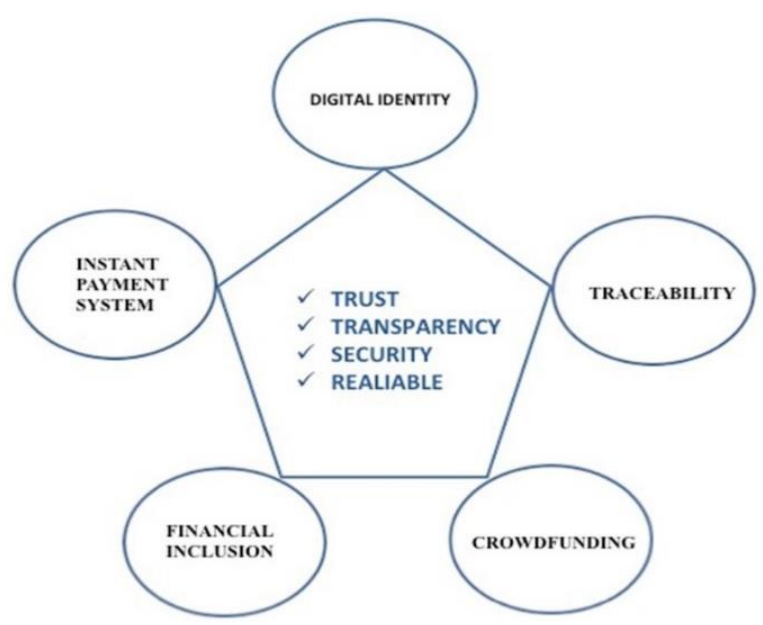

Figure 3. Blockchain and immigrants

Source: UN, 2017.

\section{Gender Equality}

From the definition of SDG5 provided by United Nations, gender equality is not only a fundamental human right, but a necessary basis for a peaceful, prosperous and sustainable world. Providing women and girls with equal access to education, medical care, decent work and representation in political and economic decisionmaking processes will drive sustainable economies and benefit societies and humanity in general (UN, 2017).

Access to women's productive resources, and among them access to financial services, energy trade, access to health services and even voting, are decisive in combating gender inequality. As determined by the World Bank, women in countries with humanitarian crises are 30 percent less likely than men to have an individual financial account. Have a safe place to store and store transfers and humanitarian remittances is a key strategy to deal with shocks and build resilience (Hammond and Young, 2018).

The Blockchain solutions that could offer more advanced financial and identification services could be the anchor in which technology could help gender equality. In this regard, we can mention specific solutions such as: IDbox, a solar-powered device which uses Blockchain to create a unique digital identity and a wallet in the absence of internet or electricity using only a $2 \mathrm{G}$ mobile phone. It was first tested in Papua New Guinea, where more than $80 \%$ of the population lacks a bank account and many remain without electricity or formal identification; or the SPENN (East Africa) case of Blockbonds that offers a digital wallet allowing users to receive, save, transfer and spend money. Encourage peer transaction interacting each other to offer goods and services, providing an opportunity to generate income. Its development aims to improve security by reducing the need for physical cash and promoting women's control over their funds, all while stimulating the financial ecosystem on the platform.

The development in this field is still in at embryonic phase, however, the potential of implementation need time to confirm if research in the field of supply chain management, land registration and identification, could generates output and practical solutions. Although to achieve theses milestones, first must be resolved about the current legal and political constrictions.

\section{Blockchain and the Management of Data Science}

Data science seeks to obtain knowledge from structured and unstructured data, ranging from statistics, data analysis, machine learning and other advanced methods with the aim of understanding and analyzing real processes from those data. The importance of trust in the data, its origin and integrity are critical for proper treatment and analysis. Among the great challenges of data science are aspects such as inaccessible data, privacy problems and dirty data. Being Blockchain, as we have already explained, a distributed digital book, there are interesting points of intersection between both fields, which could disrupt and allow correcting inefficiencies in the management of data science (Sarikaya, 2019).

In the area of data control, dirty or incorrect data, Blockchain has great potential because through the decentralized consensus algorithm and cryptography, Blockchain validates the data making it very difficult to manipulate the information. In this sense, it provides trust, transparency and privacy, essential 
characteristics to have "quality data" from which to perform analyzes and predictions. Blockchain represents a new way of managing data, a way that does not depend on a centralized file that is more easily manipulated, but that information is stored in a decentralized way, hindering possible attacks and allowing the possibility of directly analyzing data from individual devices (nodes). The data provided by Blockchain is structured, complete, immutable data and its origin can be verified through the linked chain. Another feature provided is obtaining real-time data and due to the quality provided by the cryptographic verifications of its consensus mechanism, it facilitates rapid decision making and allows blocking a suspicious transaction or tracking abnormal activities.

Data scientists can find two important opportunities in data management. On the one hand they can use the computing potential of several thousand computers connected in the blockchain network as a cloudbased service to analyze social results on a scale that is difficult to achieve otherwise. On the other hand, it allows them to manage the exchange of data, not repeating analyzes performed by other teams and avoiding erroneously performing data already used while allowing them to monetize their work, exchanging analysis results stored on the platform. However, to scale up these opportunities, however, it will be necessary to resolve the inefficiencies (energy consumption and cost) that this technology still presents in its early stage of development.

\section{Threats}

The bibliographic reviewed highlight within the most important threats that this technology must face, the regulatory challenges and the cultural issues (Stanford Graduate School of Business Blockchain for Social Impact, 2019).

Regulators look at this technology from the lack of knowledge and lack of analysis of the disruptive impact potential that would have its application, finding the developers, in a numerous cases, with hostile regulatory environments, not clear well-established regulations or total absence of them, which does not generate confidence in their development. (Irrera, 2017) As examples of frustrated developments, we can mention the case of CME Group, UK's Royal Mint a Blockchain-based gold trading platform, (Hobson, 2018) which had to freeze plans to launch a digital gold token after to the government veto to the plan for the tokens to be exchanged in a cryptocurrency exchange. In the same way, Libra currency is seeing how governments on both sides of the Atlantic try to prevent its development (Casey, 2019).

Other problems pointed out by its developers include the education of the public and the interested parties, a logic restriction, due to fact that to scaling a technology in needed look too, about customer perspective, think about willing to pay of customer, although the use of token represent a powerful tool rewarding users, but by itself it will not have the ability to break the barriers of innovation. The user experience should facilitate scalability and in this sense policy reform could lower the threshold for Blockchain-based innovation in the social impact space, being an early stage technology and taking into account that technological changes need the adaptation of the entire ecosystem.

Governments and companies around the world are investing in research and development of quantum technologies, which has significant potential to interrupt in numerous fields, including in the Blockchain scope, due to its ability to break up asymmetric cryptography (couples generation public key-private key) used (Allende, 2019). Some researchers estimate that it is possible there will be quantum computers capable of breaking cryptography in less than 10 minutes by 2027. However, solutions with more sophisticated algorithms or the development of quantum symmetric cryptography seem to be possible solutions to this threat.

\section{Conclusions}

Blockchain, being a distributed, immutable, reliable digital registry and due to its decentralization, democratization and building characteristics, offers great disruptive potential on numerous fields. Decentralization facilitates more efficient asset operations, redistributes the market power of intermediaries. Democratization favours peer exchange, increasing market dynamism. Digitization increases traceability, increasing the transparency.

All of the above opens the way for new innovations and collaboration between agencies was not possible before, as a result of the development of extensive margins. In addition to increasing efficiency, reducing verification costs, it could also mean more privacy and security, preventing data leakage. 
One of the most important applications is to facilitate digital identities. It allows access to digital identities and other essential documents providing greater transparency and therefore also greater responsibility to users, avoiding situations of abuse of power. Creating instant payment methods and facilitating financial inclusion, offering more advanced financial services, allowing the crowdfunding process to be transparent, secure and accessible by being publicly verifiable by third parties.

The use of token encourages users to make efficient and positive decisions, while providing liquidity and new interactions within the ecosystem. The use of programmable coins offers the opportunity to align individual interest with broader and holistic community interests. They could allow more control instruments to the Central Banks at the level of Monetary Policy. There are many fields that may be potentially affected by the potential of smart contracts: voting, management, supply chains, medical care and real estate. However, the inherent risks of its use should be first managed.

The research outcome can conclude that Blockchain can be a very useful tool to fight against current socioeconomic challenges, including climate change, the inclusion of disadvantaged groups, gender equality, and improvement of health services or the possibility to create innovations from data science. However, to develop its full disruptive potential serious inefficiencies must have been solved, such as the massive use of energy, which prevent its scalability. In addition, it must face regulatory challenges, overcome the threats of quantum computing and, above all, break the cultural barriers to entry of the entire ecosystem.

\section{Critims}

While the results of the research can be useful for create a framework, at a later stage, is needed a necessary empirical cost-benefit analysis, specific and customized from each specific application and in each of the solutions. So, more in deep analysis must be carries out to confirm the output of this research.

From the analysis of the existing bibliography, we indicate as a reference method, the model proposed by Panupard (2019) in his paper Cost-benefit Analysis of a Blockchain-based Supply Chain Finance Solution, especially relevant, due the fact that the exchanges carried out within the blockchain ecosystems usually use a token or cryptocurrency as an exchange unit.

\section{References}

1. Allende (2019). The road to quantum blockchain. Retrieved on $12^{\text {th }}$ September 2019 from: https://www.blockchaineconomia.es/blockchain-cuantica/.

2. Allende and Colina (2018). Blockchain: How to Develop Trust in Complex Surroundings to Generate Social Impact Value. Retrieved on $8^{\text {th }}$ November 2019 from: https://publications.iadb.org/en/blockchainhow-develop-trust-complex-surroundings-generate-social-impact-value. http://dx.doi.org/10.18235/0001139

3. Al-Saqafa and Seidlerb (2017). Blockchain technology for social impact: opportunities and challenges. $\begin{array}{lllll}\text { Retrieved on } & 5^{\text {th }} & \text { October } & 2019 & \text { from: }\end{array}$ https://www.intgovforum.org/multilingual/sites/default/files/webform/blockchaintechnologyforsocialim pact opportunitiesandchallengesahead.pdf. DOI: http://doi.org/10.1080/23738871.2017.1400084

4. Apap and Al. (2019). The Migration Issue. Retrieved on $23^{\text {th }}$ October 2019 from: http://www.europarl.europa.eu/RegData/etudes/BRIE/2019/635542/EPRS BRI(2019)635542 EN.pdf.

5. Baliga (2017). Understanding Blockchain Consensus Models. Retrieved on $2^{\text {nd }}$ September 2019 from: https://pdfs.semanticscholar.org/da8a/37b10bc1521a4d3de925d7ebc44bb606d740.pdf.

6. Barrera (2019). MIT Sloan Blockchain Tecnologies_Business Innovation and Application. Retrieved on $1^{\text {st }}$ September 2019 from: https://mitsloan.mit.edu.

7. Born (2018). Distributed Ledger Technology (DTL) for Climate Action Assessment, 2018 report. Retrieved on $7^{\text {th }}$ October 2019 from: https://www.climate-kic.org/wp-content/uploads/2018/11/DLT-forClimate-Action-Assessment-Nov-2018.pdf.

8. Cabrera (2018). United Nations blockchain pioneer prevents fraud of international aid payments. Retrieved on $23^{\text {rd }}$ September 2019 from: https://www.cio.com/article/3262791/united-nationsblockchain-pioneer-prevents-fraud-of-international-aid-payments.html.

9. Casey (2019). Article: It's Too Soon to Write Libra's Crypto Obituary. Retrieved on $19^{\text {th }}$ September 2019 from: https://www.coindesk.com/its-not-time-to-write-libras-crypto-obituary-just-yet. 
10. Carles (2019). La amenaza del cambio climático by The Menéndez Pelayo International University (UIMP). Retrieved on $5^{\text {th }}$ October 2019 from: http://www.uimp.es/actualidad-uimp/la-peor-amenaza-quetiene-la-humanidad-es-el-cambio-climatico-segun-la-fundacion-empresa-y-clima.html.

11. Church (2017). Blockchain, explained. Retrieved on $28^{\text {th }}$ August 2019 from: https://mitsloan.mit.edu/ideas-made-to-matter/blockchain-explained.

12. Cambridge Bitcoin Electricity Consumption Index (CBECI) (2019). Retrieved on $2^{\text {nd }}$ September 2019 from: https://www.cbeci.org/comparisons/.

13. Catalini (2019). MIT Sloan Blockchain Tecnologies_Business Innovation and Application. Module 3 Unit 1 Video 3 Transcript. Retrieved on $1^{\text {st }}$ September 2019.

14. Catalini and Gans (2018). Some Simple Economics of Blockchain. Working Paper 22952. Retrieved on $29^{\text {th }}$ August 2019 from: https://www.nber.org/papers/w22952 DOI: 10.3386/w22952.

15. United Nations High Commissioner for Refugees (2019). Global Trends Forced displacement in 2018 Report. Retrieved on $4^{\text {th }}$ November 2019 from: https://www.unhcr.org/5d08d7ee7.pdf.

16. Hammond and Young (2018). Can blockchain disrupt gender inequality? Retrieved on $6^{\text {th }}$ November 2019 from: https://blogs.worldbank.org/psd/can-blockchain-disrupt-gender-inequality.

17. Hobson (2018). Wary of crypto, UK government blocks Royal Mint's digital gold. Retrieved on $30^{\text {th }}$ September 2019 from: https://www.reuters.com/article/us-gold-cryptocurrency-royal-mint/wary-ofcrypto-uk-government-blocks-royal-mints-digital-gold-idUSKCN1MZ1SZ.

18. Irrera (2017). CME Group, UK's Royal Mint test blockchain-based gold trading platform. Retrieved on $18^{\text {th }}$ October 2019 on: https://www.reuters.com/article/us-blockchain-gold/cme-group-uks-royal-minttest-blockchain-based-gold-trading-platform-idUSKBN17D2RN.

19. Issberner and Léna (2018). Anthropocene: the vital problem of a scientific debate. UNESCO. Retrieved on $26^{\text {th }}$ October 2019 from: https://unesdoc.unesco.org/ark:/48223/pf0000261901.

20. Majer (2019). Non-fungible Tokens. Retrieved on $2^{\text {nd }}$ November 2019 from: https://www.blockchainresearchinstitute.org/project/non-fungible-tokens/.

21. Michelman (2017). Retrieved on $3^{\text {rd }}$ November 2019 from: https://sloanreview.mit.edu/article/seeingbeyond-the-blockchain-hype.

22. Panupard (2019). Cost-benefit Analysis of a Blockchain-based Supply Chain Finance Solution. Retrieved on $25^{\text {th }}$ September 2019 from: https://ctl.mit.edu/pub/thesis/cost-benefit-analysis-blockchain-basedsupply-chain-finance-solution.

23. Petersson (2018). How Smart Contracts Started And Where They Are Heading. Retrieved on $25^{\text {th }}$ October 2019 from: https://www.forbes.com/sites/davidpetersson/2018/10/24/how-smart-contracts-started-andwhere-they-are-heading/\#5553704a37b6.

24. Sarikaya (2019). How Blockchain Will Disrupt Data Science: 5 Blockchain Use Cases in Big Data. Retrieved on $17^{\text {th }}$ October 2019 from: https://towardsdatascience.com/how-blockchain-will-disrupt-datascience-5-blockchain-use-cases-in-big-data-e2e254e3e0ab.

25. Stanford Graduate School of Business (2019). Blockchain for Social Impact. Retrieved on $8^{\text {th }}$ October 2019 from: https://www.gsb.stanford.edu/faculty-research/publications/2019-blockchain-social-impact.

26. Tapscott and Tapscott (2016). Blockchain Revolution: How the Technology Behind Bitcoin Is Changing Money, Business, and the World. Retrieved on $10^{\text {th }}$ October 2019. New York: Penguin Random House.

27. United Nations, General Assembly, Resolution adopted by the General Assembly on $6^{\text {th }}$ July 2017. Retrieved on $4^{\text {th }}$ September 2019 from: https://undocs.org/A/RES/71/313.

28. United Nations (2017). Goal 5: Achieve gender equality and empower all women and girls. Retrieved on $2^{\text {nd }}$ October 2019 from: https://www.un.org/sustainabledevelopment/gender-equality/.

29. Wang, Wang et al. (2018). A Summary of Research on Blockchain in the Field of Intellectual Property.

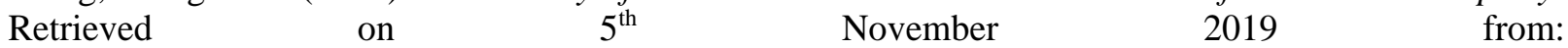
https://www.sciencedirect.com/science/article/pii/S187705091930239X. https://doi.org/10.1016/j.procs.2019.01.220. 